\title{
Time Dependent Gaussian Equivalent Linearization of Duffing Oscillator Using Continuous Wavelet Transform
}

\author{
Arunasis Chakraborty ${ }^{1}$, Prateek Mittal ${ }^{2}$ and Sabarethinam Kameshwar ${ }^{3}$ \\ 1. Department of Civil Engineering, Indian Institute of Technology Guwahati, Guwahati 781039, India \\ 2. Department of Urban and Regional Planning, University of Illinois at Urbana-Champaign, Urbana IL 61801, USA \\ 3. Department of Civil Engineering, Rice University, Houston TX 77251, USA
}

\begin{abstract}
Evolutionary response analysis of Duffing oscillator using Gaussian equivalent linearization in wavelet based time-frequency frame work is presented here. Cubic (i.e., odd type) non-linearity associated with stiffness and damping is modeled. The goal of this research is to develop the mathematical model of an equivalent linear system which is applicable for different non-stationary input processes (i.e., either summation of amplitude modulated stationary orthogonal processes or digitally simulated non-stationary processes). The instantaneous parameters of the ELTVS (equivalent linear time varying system) are evaluated by minimizing the error between the displacements of non-linear and equivalent linear systems in wavelet domain. For this purpose, three different basis functions (i.e., Mexican Hat, Morlet and a modified form of Littlewood-Paley) are used. The unknown parameters (i.e., natural frequency and damping) of the ELTVS are optimized in stochastic least square sense. Numerical results are presented for different types of input to show the applicability and accuracy of the proposed wavelet based linearization technique.
\end{abstract}

Key words: Statistical linearization, wavelet analysis, Duffing oscillator, evolutionary process.

\section{Introduction}

Stochastic response characterization of both linear and nonlinear dynamical systems has remained an active area of research in the last few decades. While theories of stationary random vibration analysis of linear system are well developed [1-3], nonlinear random vibration analysis offers several challenges and hence remains on open area of research. Caughey $[4,5]$ developed mathematical models for linear and nonlinear systems based on the theory of Markov processes. In these studies, the systems were subjected to white noise excitation and the response was evaluated by solving the FPK (Fokker-Planck-Kolmogrov) equation. Hammond [6] used evolutionary spectral representation of a non-stationary process to study the response of a linear oscillator. Orabi [7] developed nonstationary response

Corresponding author: Arunasis Chakraborty, Ph.D., assistant professor, research fields: earthquake engineering, random vibrations and wavelet analysis. E-mail: arunasis@iitg.ernet.in. analysis of nonlinear systems using the functional series expansion method. Recently, Cross et al. [8] developed approximate solution for frequency response function of Duffing oscillator using FPK equations to model higher order resonance. Here, it may be mentioned that close-form solutions based on FPK equations are limited to certain class of input. For nonlinear systems, equivalent linearization is the most popular tool for response characterization.

In equivalent linearization, the nonlinear system is replaced by an equivalent linear system whose parameters are evaluated in stochastic least square sense [9, 10]. Caughey [11] developed approximate solution for weakly nonlinear systems using equivalent linearization. He considered nonlinearity associated with velocity and displacement. Iwan et al. [12] used equivalent linearization to solve the response of nonlinear system under nonstationary excitations. Iyengar [13] developed analytical model for the stochastic response of Duffing oscillator excited by white noise. A comprehensive review of statistical 
linearization may be found in the book by Robert et al. [14]. Elishakoff et al. [15] and Elishakoff [16] suggested the use of both potential energy and complementary energy as the criteria for linearization. Sobiechowski et al. [17] derived expression response of Duffing oscillator when the input is non Gaussian. Pradlwater [18] used local linearization coupled with Gaussian superposition to estimate the probability density function of a nonlinear system. Mickens [19] used combined equivalent linearization and perturbation technique to solve the response of nonlinear systems. Crandall [9] used non Gaussian input for statistical linearization of Duffing oscillator and other oscillators. Roy et al. [20] generalized locally transversal linearization and developed multi step transversal linearization method for solving nonlinear oscillators. Lacquaniti et al. [21] proposed probabilistic linearization based on FPK equations for nonlinear systems subjected to additive and multiplicative random excitations. Li et al. [22] used equivalent linearization based on equivalence of energy dissipated by two systems. In view of these models for random vibration analysis, it may be mentioned that conventional techniques often face difficulty to model both amplitude and frequency nonstationarity. For this purpose, wavelet based time-frequency analysis has gained popularity in the recent past. Basu et al. [23-26] and Chakraborty et al. [27] used wavelet based time-frequency analysis to evaluate the response of randomly excited linear and nonlinear systems. Wavelet transform has time localization property which makes it more suitable when the input has time varying amplitude and frequency content. Spanos et al. [28] used different wavelet basis functions to model the nonstationary process. From these studies, one may conclude that stochastic response analysis of nonlinear systems especially in the light of both amplitude and frequency nonstationary offers several challenges and needs further attention.

With this in view, present work aims to develop wavelet based equivalent linearization technique for a
Duffing oscillator subjected to nonstationary random process. The time localization characteristics of the wavelet transform is proposed to be utilized to capture the fluctuation of amplitude and frequency content of the input signal to evaluate the instantaneous parameters of the time varying equivalent linear system.

\section{Problem Formulations}

In this paper, the Duffing oscillator shown in Fig. 1 is considered for evolutionary response analysis. The governing equation of motion is given by:

$$
\ddot{x}+g(x, \dot{x})=-\ddot{x}_{g}
$$

The time function $\ddot{x}_{g}(t)$ in the above equation represents the nonstationary acceleration at the support. Function $g(x, \dot{x})$ in Eq. (1) is given by:

$$
g(x, \dot{x})=2 \eta \omega_{n} \dot{x}+\lambda_{1} 2 \eta \omega_{n} \dot{x}^{3}+\omega_{n}^{2} x+\lambda_{2} \omega_{n}^{2}
$$

Parameters $\eta$ and $\omega_{n}$ are the damping ratio and un-damped natural frequency of the linear system. Parameter $\lambda_{1}$ and $\lambda_{2}$ in Eq. (2) control the degree of nonlinearity. The nonlinear part of the stiffness and damping varies with the cubic power of the displacement and velocity, respectively. Using statistical linearization, the Duffing oscillator as described in Eq. (1) is replaced by an equivalent linear system whose governing equation is given by

$$
\ddot{x}+2 \eta_{e q} \omega_{e q} \dot{x}+\omega_{e q}^{2} x=-\ddot{x}_{g}
$$

In the above equation, $\eta_{e q}$ and $\omega_{e q}$ are the damping ratio and un-damped natural frequency of the equivalent

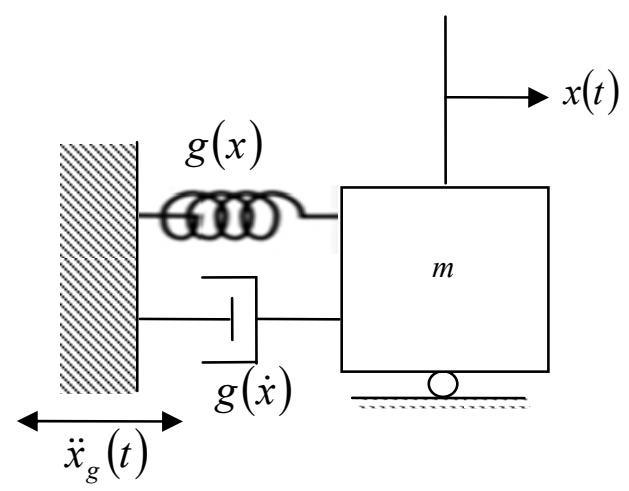

Fig. 1 Duffing oscillator. 
linear system. For simplifying mathematical operations, a new parameter, $\overline{\eta_{e q}}=\eta_{e q} \omega_{e q}$ is introduced. Hence the equation of motion for equivalent linear system takes the form:

$$
\ddot{x}+2 \overline{\eta_{e q}} \dot{x}+\omega_{e q}^{2} x=-\ddot{x}_{g}
$$

The unknown parameters in the above equation are evaluated in least square sense by minimizing the expected value of the square of the error between Eq. (1) and Eq. (4). However, as the input process is assumed to be non-stationary (whose exact mathematical model may or may not be known), the unknown parameters in Eq. (4) are bound to vary with time. In view of this non-stationary nature, the time-frequency localization characteristics of wavelet based signal processing is proposed to be utilized here to evaluate the unknown time varying parameters in Eq. (4).

\subsection{Brief Overview of Continuous Wavelet Transform}

Wavelet transform has been widely used for different applications of signal processing in the recent past. Plenty of books and literatures [1, 29, 30] are available that describe wavelet transform and its applications in details. Here, a brief overview of the wavelet transform is presented for continuity. Let $f(t)$ be a square integrable function in $L^{2}(R)$ (i.e., $f^{2}(t) d t<$ $\infty)$, then using continuous wavelet transform $f(t)$ can be converted into two dimension with parameters " $a$ " and " $b$ ", which is given by:

$$
W_{\psi} f(a, b)=\int_{-\infty}^{\infty} f(t) \psi_{a, b}^{*}(t) d t
$$

where, “*” denotes the complex conjugate and $\psi_{a, b}^{*}(t)$ is the dilated and time localized version of the mother wavelet $\Psi(t)$ :

$$
\psi_{a, b}^{*}=\frac{1}{\sqrt{|a|}} \psi^{*}\left(\frac{t-b}{a}\right)
$$

Parameter " $a$ " dilates the wavelet basis and provides frequency resolution while parameter " $b$ " localizes it in time (i.e., around $t=b$ ). The original time signal $f(t)$ can be traced back from the wavelet coefficients using the following relationship:

$f(t)=\frac{1}{2 \pi C_{\psi}} \int_{-\infty}^{\infty} \int_{-\infty}^{\infty} \frac{1}{a^{2}} W_{\psi} f(a, b) \psi\left(\frac{t-b}{a}\right) \frac{d a}{\sqrt{|a|}} d b(7)$

Parameter $\mathrm{C}_{\Psi}$ in the above equation is the admissibility criteria for $\Psi(t)$ to be a wavelet basis and is given by:

$$
C_{\psi}=\int_{-\infty}^{\infty} \frac{|\Psi(\omega)|^{2}}{|\omega|} d \omega<\infty
$$

In the above equation, $\Psi(\omega)$ is the Fourier transform of wavelet basis $\Psi(t)$. It may be noticed that parameters " $a$ " and " $b "$ are continuous. However, following discretization scheme is adopted for parameters " $a$ " and " $b$ " in the present study for the purpose of numerical analysis:

$$
\left\{\begin{array}{c}
a_{j}=\sigma^{j} \\
b_{i}=(i-1) \Delta b
\end{array}\right\}
$$

It can be shown that the above discretization scheme reduces Eq. (7) to the following form:

$$
f(t)=\sum_{j} \sum_{i} \frac{K \Delta b}{a_{j}} W_{\psi}\left(a_{j}, b_{i}\right) \psi\left(\frac{t-b_{i}}{a_{j}}\right)
$$

The constant " $K$ " in Eq. (10) is given by:

$$
K=\left(\sigma^{2}-1\right) / 4 \pi C_{\psi} \sigma
$$

In this context, selection of wavelet basis is an important step as it affects the frequency and time resolution of the wavelet transform. In this paper, three different basis functions are used to evaluate the wavelet coefficients in Eq. (5).

(1) Modified form of little-wood Paley basis function (MLP)

This basis function is constructed using the real part of the harmonic wavelet basis [1]. The time and frequency signature of this basis are given by:

$$
\left\{\begin{array}{l}
\psi(t)=\frac{1}{\pi \sqrt{\sigma-1}} \frac{\sin (\sigma \pi t)-\sin (\pi t)}{t} \\
\psi(\omega)=\frac{1}{\sqrt{2(\sigma-1) \pi}} \quad \pi \leq|\omega| \leq \sigma \pi
\end{array}\right\}
$$

From the above expression, one can notice that $\Psi(\omega)$ has compact support in frequency domain;

(2) Mexican Hat basis function (Mex)

The time and frequency description of the Mexican Hat basis function is given by:

$$
\left\{\begin{array}{l}
\psi(t)=\frac{2}{\sqrt{3} \sqrt[4]{\pi}}\left(1-t^{2}\right) e^{-t^{2} / 2} \\
\psi(\omega)=\frac{2}{\sqrt{3} \sqrt[4]{\pi}}\left(\omega^{2}\right) e^{-\omega^{2} / 2}
\end{array}\right\}
$$


The time and frequency description of the Morlet basis function is given by:

$$
\left\{\begin{array}{c}
\psi(t)=e^{-t^{2} / 2} \cos \left(\omega_{c} t\right) \\
\psi(\omega)=\frac{e^{\left(-\omega^{2}-\omega_{c}^{2}\right)}}{2}\left(e^{\left(\omega-\omega_{c}\right)^{2} / 2}+e^{\left(\omega+\omega_{c}\right)^{2} / 2}\right)
\end{array}\right\}
$$

Parameter $\omega_{c}$ in the above equation represents the central frequency of this basis and is assumed to be 5 $\mathrm{rad} / \mathrm{s}$ throughout this paper.

\subsection{Non-stationary Input in Wavelet Domain}

In this paper, the support motion is modeled as amplitude modulated stationary process which is mathematically expressed as Refs. [31, 32]:

$$
\ddot{x}_{g}=\sum_{r=1}^{n} \int_{-\infty}^{\infty} A_{r}(\omega, t) e^{i \omega t} d G_{r}(\omega)
$$

where, $A_{r}(\omega, t)$ represents the $r^{\text {th }}$ amplitude modulation function dependent on time and frequency. In above equation, $d G_{r}(\omega)$ presents the $r^{\text {th }}$ orthogonal stationary Gaussian process which is given by:

$$
\begin{aligned}
E\left[d G_{r}(\omega) \cdot d G_{r}^{*}\left(\omega^{\prime}\right)\right] & =S_{f f}^{r}(\omega) d \omega ; \omega=\omega^{\prime} \\
& =0 \quad 0 \quad ; \omega \neq \omega^{\prime}
\end{aligned}
$$

Taking wavelet transform of both sides of Eq. (15), it can be shown that wavelet coefficients of $\ddot{x}_{g}(t)$ can be expressed as Ref. [28]:

$$
W_{\psi} \ddot{x}_{g}\left(a_{j}, b\right)=\sum_{r=1}^{n} \int_{-\infty}^{\infty} A_{r}(\omega, b) e^{i \omega b} d \tilde{G}_{r}(\omega)(17)
$$

In Eq. (17), the frequency parameter has been discretized and hence $a_{j}$ represents the $j^{\text {th }}$ frequency scale while the time parameter " $b$ " is kept continuous. It can be seen that the wavelet coefficients $W_{\psi} \ddot{x}_{g}\left(a_{j}, b\right)$ at a scale $a_{j}$ has a similar mathematical expression as that of $\ddot{x}_{g}(t)$. The orthogonal process in wavelet domain can be shown as:

$$
\begin{array}{rlr}
E\left[d \tilde{G}_{r}(\omega) \cdot d \tilde{G}_{r}^{*}\left(\omega^{\prime}\right)\right] & =\bar{S}(\omega) d \omega ; \omega=\omega^{\prime} \\
& = & 0 ; \omega \neq \omega^{\prime}
\end{array}
$$

where, $\bar{S}(\omega)=2 \pi a_{j}\left|\Psi\left(\omega a_{j}\right)\right|^{2} S_{f f}^{r}(\omega)$. In the above equation, $\Psi\left(\omega a_{j}\right)$ represents the Fourier transform of the wavelet basis function at the $j^{\text {th }}$ frequency scale $a_{j}$.

In this study, two different examples of the input processes are considered. In the first example, the system is excited by an amplitude modulated stationary process. The amplitude modulation $A(t)$ and the stationary processes $S_{f f}(\omega)$ are given by:

$$
\begin{gathered}
A(t)=\gamma\left(e^{-\alpha t}-e^{-\beta t}\right) \\
S_{f f}(\omega)=S_{o}\left[\frac{4 \eta_{g}^{2}\left(\omega / \omega_{g}\right)^{2}}{\left[1-\left(\omega / \omega_{g}\right)^{2}\right]^{2}+4 \eta_{g}^{2}\left(\omega / \omega_{g}\right)^{2}}\right]
\end{gathered}
$$

In the second case, EL-Centro ground motion which is modeled as the summation of different amplitude modulated stationary orthogonal process is used. The envelop function and the power spectral density for the recorded El-Centro ground motions as suggested by Conte et al. [33] is used in the analysis and is expressed as:

$$
\begin{aligned}
& A_{r}(t)=\alpha_{r}\left(t-\zeta_{r}\right)^{\beta_{r}} e^{-\gamma_{r}\left(t-\zeta_{r}\right)} H\left(t-\zeta_{r}\right) \\
& S_{f f}(\omega)=\frac{v_{r}}{2 \pi}\left[\frac{1}{v_{r}^{2}+\left(\omega+\eta_{r}\right)^{2}}+\frac{1}{v_{r}^{2}+\left(\omega-\eta_{r}\right)^{2}}\right]
\end{aligned}
$$

For brevity, the detailed description of the terms in Eqs. (21) and (22) are omitted here. The reader may refer to Conte et al. [33] for further details.

\subsection{Wavelet Based Linearization}

In this section, a LTVS (linear time varying system) is designed in wavelet domain in such a way that the error between the Duffing oscillator and the LTVS is minimized with respect to its parameters in stochastic sense. The first step in this process is to express the governing equations in wavelet domain. On transforming both sides of Eq. (1) in wavelet domain, one can show that:

$$
\frac{\partial^{2}}{\partial b^{2}} W_{\psi} x\left(a_{j}, b\right)+W_{\psi} g\left(a_{j}, b\right)=W_{\psi} \ddot{x}_{g}\left(a_{j}, b\right)
$$

Similarly, wavelet transforming both sides of Eq. (3) one gets:

$$
\begin{gathered}
\frac{\partial^{2}}{\partial b^{2}} W_{\psi} x\left(a_{j}, b\right)+2 \overline{\eta_{e q}} \frac{\partial}{\partial b} W_{\psi} x\left(a_{j}, b\right)+ \\
\omega_{e q}^{2} W_{\psi} x\left(a_{j}, b\right)=W_{\psi} \ddot{x}_{g}\left(a_{j}, b\right)
\end{gathered}
$$

It can be seen that $\omega_{e q}$ and $\overline{\eta_{e q}}$ are the unknowns in the above equation. The difference between Eqs. (23) and (24) represents the error. On taking expectation of the square of this error at $i^{\text {th }}$ time instant and $j^{\text {th }}$ scale, one can show that:

$$
\begin{gathered}
E\left[\varepsilon_{i j}^{2}\right]=E\left[\left\{2 \overline{\eta_{e q}} W_{\psi} \dot{x}\left(a_{j}, b_{i}\right)+\omega_{e q}^{2} W_{\psi} x\left(a_{j}, b_{i}\right)-\right.\right. \\
\left.\left.W_{\psi} g\left(a_{j}, b_{i}\right)\right\}^{2}\right]
\end{gathered}
$$

The gross error at $i^{\text {th }}$ time instant (i.e., for all $j$ ) in Eq. (25) is differentiated with respect to the unknown 
parameters $\omega_{e q}^{2}$ and $\overline{\eta_{e q}}$ to form two simultaneous equations which is given by:

$$
\begin{gathered}
\frac{\partial}{\partial \omega_{e q}^{2}} \sum_{j} \frac{1}{a_{j}} E\left[\varepsilon_{i j}^{2}\right]=0 \\
\frac{\partial}{\partial \bar{\eta}_{e q}} \sum_{j} \frac{1}{a_{j}} E\left[\varepsilon_{i j}^{2}\right]=0
\end{gathered}
$$

On simplifying above expression and applying properties of wavelet transform and Gaussian process, it can be proved that:

$$
\begin{aligned}
& \omega_{e q_{i}}^{2}=\omega_{n}^{2}\left[1+3 \lambda_{2} \sum_{j} \frac{K}{a_{j}} E\left[W_{\psi}^{2} x\left(a_{j}, b_{i}\right)\right]\right] \\
& \overline{\eta_{e q_{l}}}=\eta \omega_{n}\left[1+3 \lambda_{1} \sum_{j} \frac{K}{a_{j}} E\left[W_{\psi}^{2} \dot{x}\left(a_{j}, b_{i}\right)\right]\right]
\end{aligned}
$$

From Eqs. (28) and (29), it can be noticed that the solution for natural frequency and damping of the LTVS need second moment of the displacement and velocity in wavelet domain. Therefore, to solve for the unknown parameters, the evolutionary response of the LTVS system is first modeled in wavelet domain. With this in view, the response $W_{\Psi} x\left(a_{j}, b\right)$ from Eq. (24) in wavelet domain at scale $a_{j}$ may be written as:

$$
W_{\psi} x\left(a_{j}, b\right)=\int_{0}^{b} h(b-\tau) W_{\psi} \ddot{x}_{g}\left(a_{j}, \tau\right) d \tau
$$

In the above equation, $h(\cdot)$ represents the impulse response function. Substituting Eq. (17) in Eq. (30) and simplifying, one can show that

$$
\begin{aligned}
& W_{\psi} x\left(a_{j}, b\right)= \\
& \sum_{r=1}^{n} \int_{0}^{b} h(b-\tau) \int_{-\infty}^{\infty} A_{r}(\omega, \tau) e^{i \omega \tau} d \tilde{G}_{r}(\omega) d \tau \\
& \quad=\sum_{r=1}^{n} \int_{-\infty}^{\infty} M_{r}(\omega, b) e^{i \omega b} d \tilde{G}_{r}(\omega)
\end{aligned}
$$

where, $M_{r}(\omega, b)$ is given by:

$$
M_{r}(\omega, b)=\int_{0}^{b} h(b-\tau) A_{r}(\omega, \tau) e^{i \omega(\tau-b)} d \tau
$$

It is assumed that amplitude modulations in Eq. (15) are independent of frequency i.e., $A_{r}(\omega, t)=A_{r}(t)$. Moreover, it may be noticed that in Eq. (30), $A_{r}(\omega, t)$ is a slowly varying function of time as compared to $h($ ). Therefore, as the wavelet transform is localized around $t=b, A_{r}(\omega, \tau)$ may be approximated as $A_{r}(b)$ and may be considered constant. Therefore, $M_{r}(\omega, b)$ may be approximated as

$$
M_{r}(\omega, b)=-A_{r}(b) H(\omega)
$$

where, $H(\omega)$ is the frequency response function of the LTVS and is given by:

$$
H(\omega)=\frac{1}{\left(\omega_{e q i}{ }^{2}-\omega^{2}\right)+i\left(2 \eta_{e q i} \omega_{e q i} \omega\right)}
$$

Substituting Eq. (33) in Eq. (31) and multiplying both sides of Eq. (31) by its complex conjugate, one can show that:

$$
E\left[W_{\psi}^{2} x\left(a_{j}, b\right)\right]=
$$

$\sum_{r=1}^{n} 4 \pi a_{j}\left|A_{r}(b)\right|^{2} \int_{0}^{\infty}|H(\omega)|^{2}\left|\Psi\left(\omega a_{j}\right)\right|^{2} S_{f f}^{r}(\omega) d \omega$ (35)

On differentiating both sides of Eq. (31), it can be shown that:

$$
W_{\psi} \dot{x}\left(a_{j}, b\right)=\sum_{r=1}^{n} \frac{\partial}{\partial b} \int_{-\infty}^{\infty} M_{r}(\omega, b) e^{i \omega b} d \tilde{G}_{r}(\omega)
$$

Substituting the expression for $M_{r}(\omega, b)$ and performing differentiation using chain rule, one gets:

$$
\begin{gathered}
W_{\psi} \dot{x}\left(a_{j}, b\right)= \\
\sum_{r=1}^{n} \int_{-\infty}^{\infty}-A_{r}(b) H(\omega) i \omega e^{i \omega b} d \bar{G}_{r}(\omega)+ \\
\sum_{r=1}^{n} \int_{-\infty}^{\infty}-\dot{A}_{r}(b) H(\omega) e^{i \omega b} d \bar{G}_{r}(\omega)
\end{gathered}
$$

Again it may be noticed that as $A_{r}(b)$ is approximated as slowly varying function at around $t=b$, its time derivative i.e., $\dot{A}(b)$ can be neglected. Therefore, considering the first term and multiplying Eq. (37) by its complex conjugate and taking the expectation, one can show that:

$$
E\left[W_{\psi}^{2} \dot{x}\left(a_{j}, b\right)\right]=
$$

$\sum_{r=1}^{n} 4 \pi a_{j}\left|A_{r}(b)\right|^{2} \int_{0}^{\infty}|H(\omega)|^{2} \omega^{2}\left|\Psi\left(\omega a_{j}\right)\right|^{2} S_{f f}^{r}(\omega) d \omega$

Now all the expressions required to evaluate $\omega_{e q_{i}}$ and $\eta_{e q_{i}}$, from Eqs. (28) and (29), have been defined. Hence two error functions can be formulated as:

$$
\begin{gathered}
\varepsilon_{1, i}^{2}=\left[\omega_{\text {eqi }}^{2}-\omega_{n}^{2}\left[1+3 \lambda_{2} \sum_{j} \frac{K}{a_{j}} E\left[W_{\psi}^{2} x\left(a_{j}, b_{i}\right)\right]\right]\right]^{2} \\
\varepsilon_{2, i}^{2}=\left[\overline{\eta_{e q l}}-\eta \omega_{n}\left[1+3 \sum_{j} \frac{K}{a_{j}} E\left[W_{\psi}^{2} \dot{x}\left(a_{j}, b_{i}\right)\right]\right]\right]^{2}
\end{gathered}
$$

To find the parameters of the equivalent linear system, the two error functions will have to be minimized simultaneously. This can be done by defining a new error function which is the sum of squares of the original error functions:

$$
\varepsilon=\varepsilon_{1, i}^{2}+\varepsilon_{2, i}^{2}
$$

To minimize the error function expressed in Eq. (41), in-built functions of optimization toolbox in MATLAB are used. When the natural frequency and damping of LTVS is calculated, then PSDF (power spectral density function) of the displacement of the nonlinear system may be evaluated using the following relation [28]: 


$$
\bar{S}_{x x}(\omega, b)=\sum_{j=1}^{n} \alpha_{j}(b)\left|\Psi\left(\omega a_{j}\right)\right|^{2}
$$

where, $a_{j}(b)$ is evaluated from the following matrix equation at each time instant:

$$
\begin{gathered}
{\left[\begin{array}{cccc}
K_{1,1} & K_{1,2} & \ldots & K_{1, n} \\
K_{2,1} & K_{2,2} & \ldots & K_{2, n} \\
\ldots & \ldots & \ldots & \ldots \\
K_{n, 1} & K_{n, 2} & \ldots & K_{n, n}
\end{array}\right]\left[\begin{array}{c}
\alpha_{1}(b) \\
\alpha_{2}(b) \\
\ldots \\
\alpha_{n}(b)
\end{array}\right]=} \\
\\
{\left[\begin{array}{c}
E\left[W_{\psi} f\left(a_{1}, b\right)^{2}\right] \\
E\left[W_{\psi} f\left(a_{2}, b\right)^{2}\right] \\
\ldots \\
E\left[W_{\psi} f\left(a_{n}, b\right)^{2}\right]
\end{array}\right]}
\end{gathered}
$$

The coefficients of the above matrix can be evaluated from the wavelet basis function and are given by:

$$
K_{r, s}=4 \pi a_{r} \int_{0}^{\infty}\left|\Psi\left(\omega a_{r}\right)\right|^{2}\left|\Psi\left(\omega a_{s}\right)\right|^{2} d \omega
$$

Finally, the higher order moments of the response of the Duffing oscillator may be evaluated once the displacement PSDF is calculated using Eq. (42).

\section{Numerical Results and Discussion}

In this section, numerical analysis of the wavelet base equivalent linearization of Duffing oscillator is carried out. Two different example cases are solved to study the accuracy of effectiveness of the proposed method. The parameters $\lambda_{1}$ and $\lambda_{2}$ in Eq. (2) are assumed to be 0.1 throughout this numerical study. In the first example, a single amplitude modulated stationary processes as discussed in Eqs. (19) and (20) is considered. The natural frequency and the damping, i.e., $\omega_{g}$ and $\eta_{g}$ of the input process as defined in Eq. (20) are assumed to be $12 \mathrm{rad} / \mathrm{s}$ and 0.4 , respectively. The parameters of the envelop function in Eq. (19) (i.e., $\alpha$, $\beta$ and $\lambda$ ) are $0.25,0.5$ and 4 , respectively. For these set of values, the envelope function attains a peak value of 1 at $2.5 \mathrm{~s}$. The natural frequency and critical damping ratio, i.e., $\omega_{n}$ and $\eta$ in Eq. (1) are assumed to be 40 $\mathrm{rad} / \mathrm{s}$ and $5 \%$, respectively. Using these values, wavelet based statistical linearization is performed to evaluate the time varying parameters of the equivalent linear system. Three different wavelet bases are used namely
MLP, Mexhat and Morlet. The details of the parameters for these bases are listed in Table 1. Fig. 2 shows the instantaneous natural frequency and damping ratio of the equivalent LTVS. It may be noticed that the values of the equivalent parameters obtained from Eqs. (28) and (29) using three different bases match closely. Finally, the instantaneous normalized mean square values, i.e., $E\left[x_{i}^{2}\right] / \sum E\left[x_{i}^{2}\right]$ of the equivalent LTVS are compared with simulation results.

A close match is observed between them which indicate that the accuracy of the proposed wavelet based model. In this context, 100 samples of the earthquake accelarogram are used for the simulation. The wavelet based linearization model is then further used to study the stochastic response of the Duffing oscillator. Fig. 3 shows the variation of peak mean square value for different $\omega_{n}$. A close match between the wavelet results using different bases and the simulation is also observed in this case. Fig. 4 shows the instantaneous PSDF at $3.5 \mathrm{~s}$ and $7 \mathrm{~s}$. It may be noticed that as the MLP basis has compact support in frequency domain, the PSDF obtained using this basis function shows band localization. Nevertheless, it matches with the results obtained using other basis functions, i.e., Mexhat and Morlet in mean sense.

In the second example, El-Centro ground motion is used, which is modeled by Eqs. (21) and (22). For the details of this model, one may refer to Ref. [33]. The El-Centro ground motion is modeled as summation of amplitude modulated stationary orthogonal processes. The details of the parameter of the twenty one different amplitude modulations and stationary processes are

Table 1 Model parameters for wavelet transform.

\begin{tabular}{lll}
\hline Basis function & $\sigma$ & $J$ \\
\hline MLP & $2^{1 / 4}$ & -15 to 6 \\
Mex. Hat & 2 & -8 to 6 \\
Morlet & $2^{1 / 2}$ & -9 to 5 \\
\hline
\end{tabular}





$\mathrm{t}(\mathrm{s})$

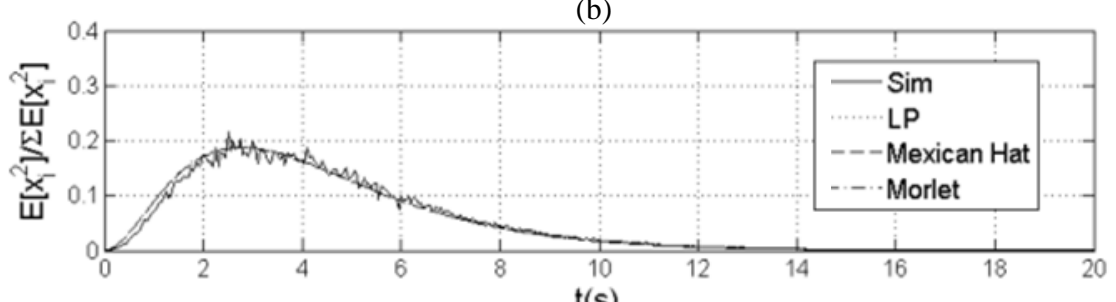

(c)

Fig. 2 Parameters of equivalent linear system for $\omega_{n}=40 \mathrm{rad} / \mathrm{s}$ and $\boldsymbol{\eta}=\mathbf{5 \%}$ under amplitude modulated stationary excitation: (a) $\omega_{e q i}$; (b) $\eta_{e q i}$; (c) normalized mean square value.

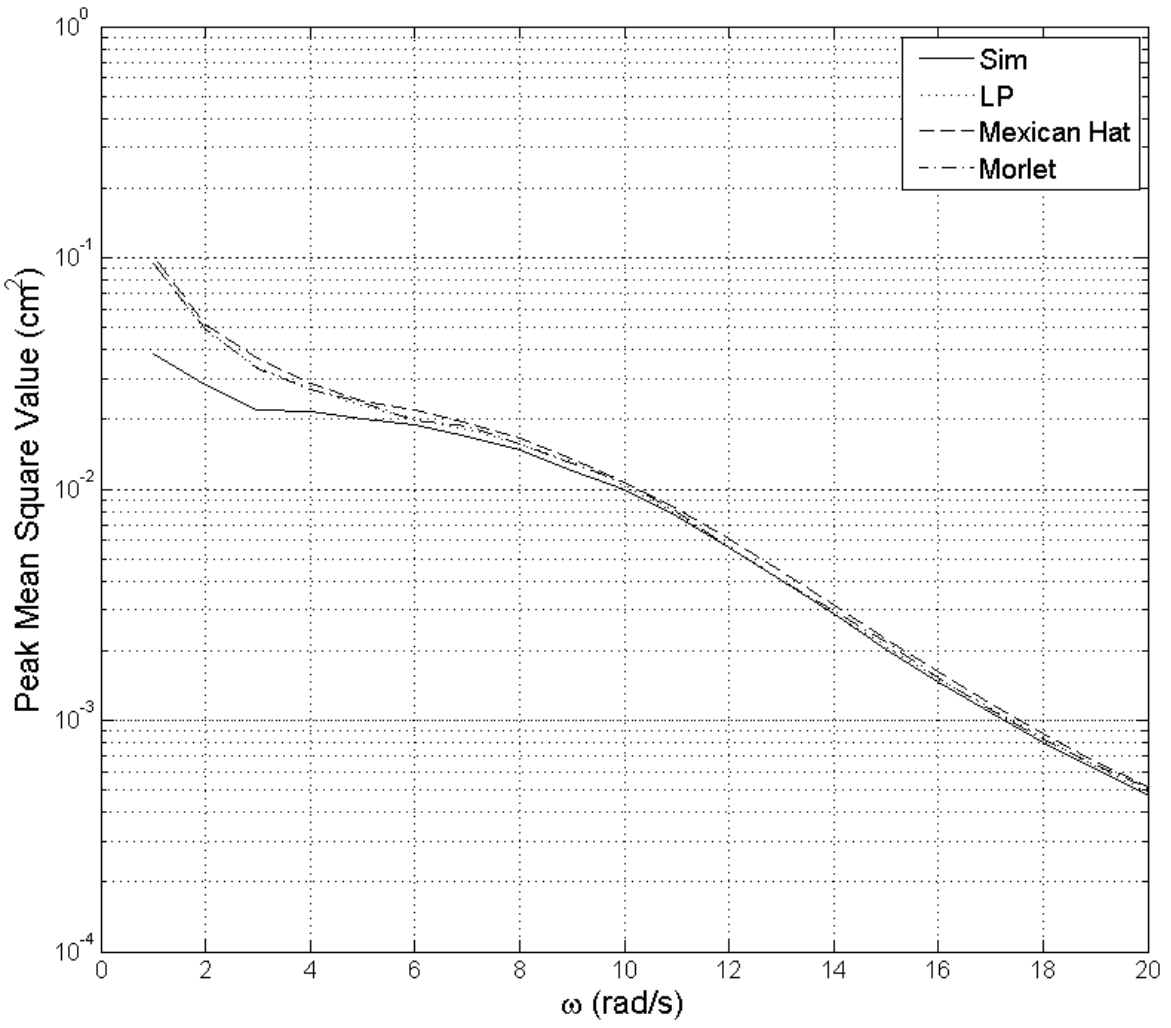

Fig. 3 Peak mean square value vs. $\omega_{n}$ for $\eta=5 \%$ for amplitude modulated stationary excitation. 

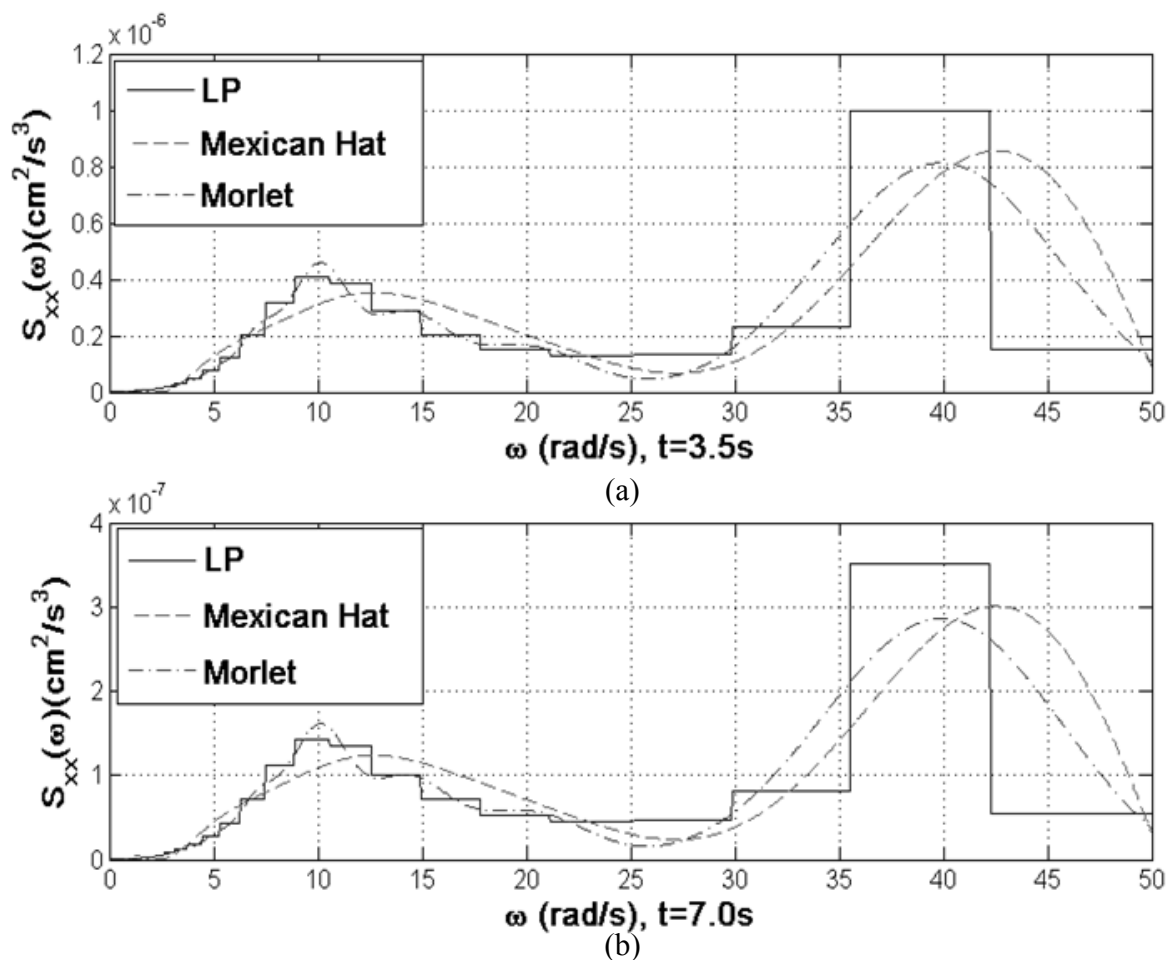

Fig. 4 PSDF of the LTVS for $\omega_{n}=40 \mathrm{rad} / \mathrm{s}$ and $\eta=5 \%$ for amplitude modulated stationary excitation: (a) at $t=3.5 \mathrm{~s}$; (b) at $t$ $=7 \mathrm{~s}$.

given in Ref. [33]. The natural frequency and damping, i.e., $\omega_{n}$ and $\eta$ are assumed to be $40 \mathrm{rad} / \mathrm{s}$ and $10 \%$, respectively.

Fig. 5 shows the instantaneous values of the natural frequency and damping of the equivalent LTVS for three different basis functions. The values of the parameters in both the cases match close for different bases. Fig. 5c shows the instantaneous normalized mean square values of the equivalent LTVS using three bases and simulation and the wavelet results closely match with the simulation which, in turn, proves the accuracy of the proposed linearization scheme. Fig. 6 shows the peak mean square value of the response for different natural frequencies. It can be observed that at high frequency, the response evaluated by three different bases closely matches with simulation results. While at low frequency range, MLP basis function provides better results.

Fig. 7 shows instantaneous PSDF at $3.5 \mathrm{~s}$ and $7 \mathrm{~s}$ for different bases. Here also the MLP basis shows step like frequency localization as it has compact support in frequency. Nevertheless, the results match with each other in average sense. Finally, Figs. 8-10 show response PSDF evaluated using three different bases. From these figures, one may easily conclude that the proposed wavelet based linearization scheme successfully models the amplitude and frequency non-stationary nature of the response of the non-linear system. In this context, it may be noticed that results obtained using MLP and Morlet bases are similar as their peak values and frequency localization match closely.

Although the peak value of the PSDF obtained by this basis function matches with other two, the frequency localization is not distinct as compared to MLP and Morlet bases. Nevertheless, the area under the PSDF at different time (i.e., instantaneous mean square values) obtained by three different bases are same, which is also reflected in Figs. 5 and 6. All these results establish that the proposed model of the wavelet based statistical linearization can be adopted for the evolutionary response analysis of the Duffing oscillator. 

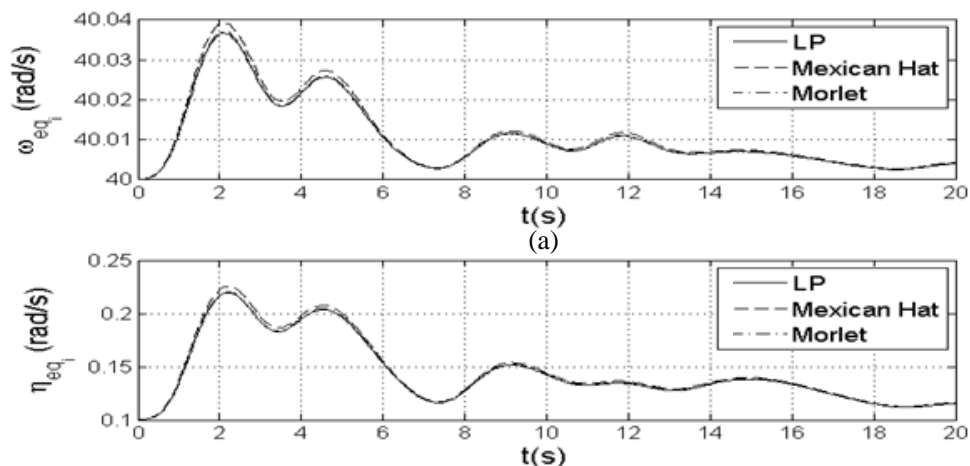

(b)

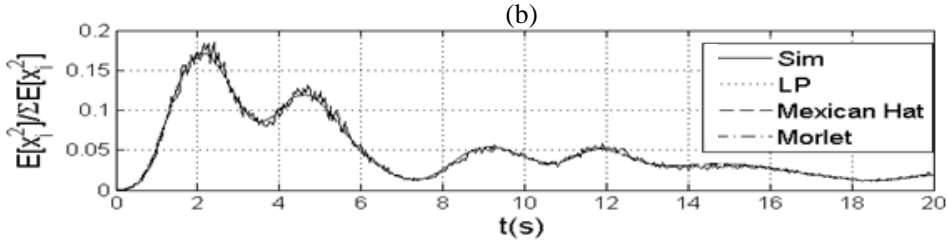

(c)

Fig. 5 Parameters of equivalent linear system for $\omega_{n}=40 \mathrm{rad} / \mathrm{s}$ and $\eta=5 \%$ under El Centro excitations: (a) $\omega_{e q i}$; (b) $\eta_{\text {eqi }}$; (c) normalized mean square value.

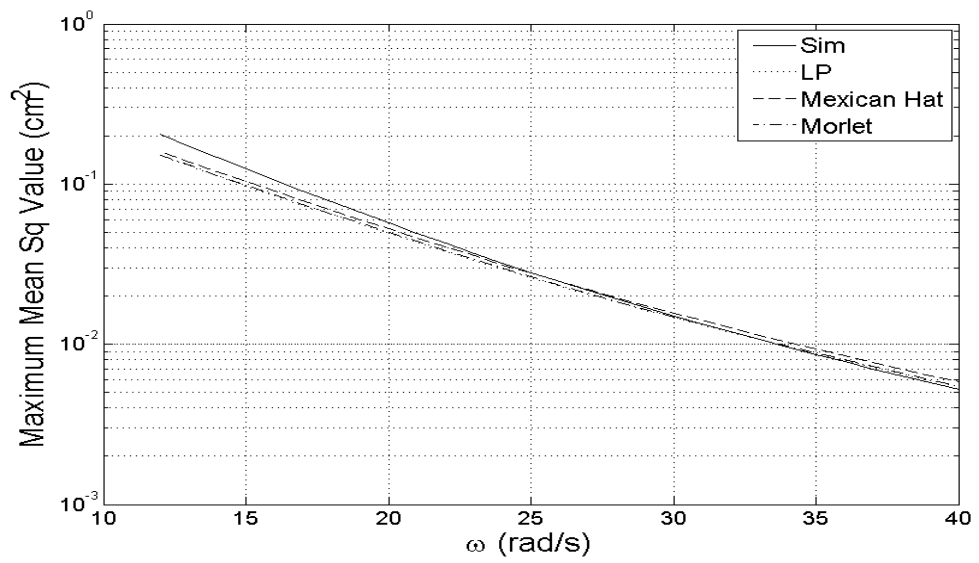

Fig. 6 Peak mean square value vs. $\omega_{n}$ for $\eta=10 \%$ for El Centro excitations.


(b)

Fig. 7 PSDF of the LTVS for $\omega_{n}=40 \mathrm{rad} / \mathrm{s}$ and $\eta=5 \%$ for El Centro excitations: (a) $t=3.5 \mathrm{~s}$; (b) at $t=7 \mathrm{~s}$. 


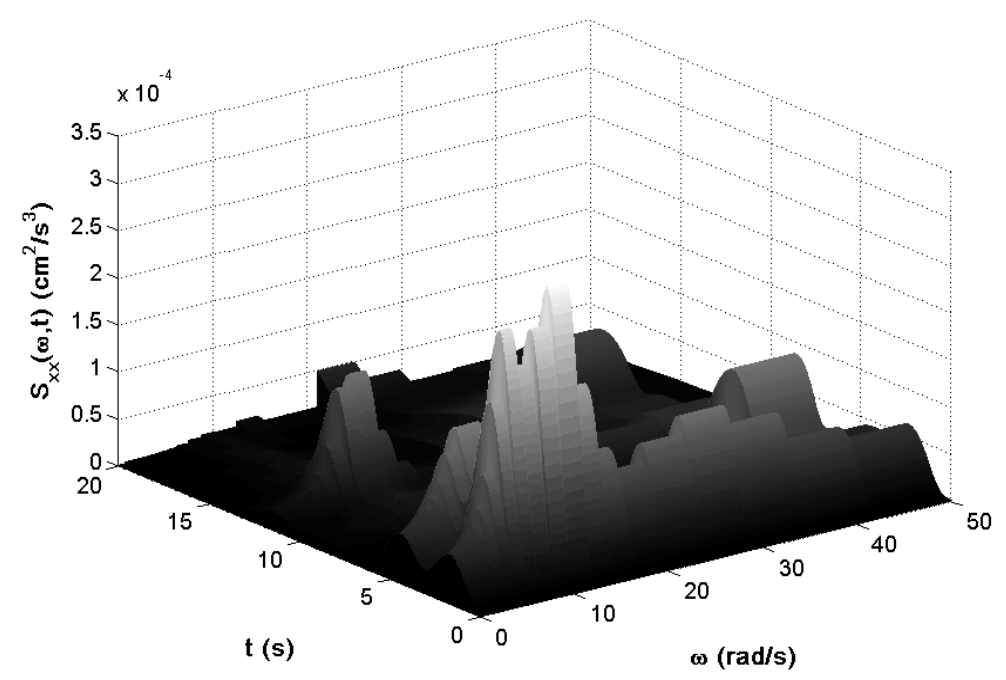

Fig. 8 Displacement PSDF using LP basis function for $\omega_{n}=40 \mathrm{rad} / \mathrm{s}$ and $\eta=10 \%$ for EI Centro excitations.

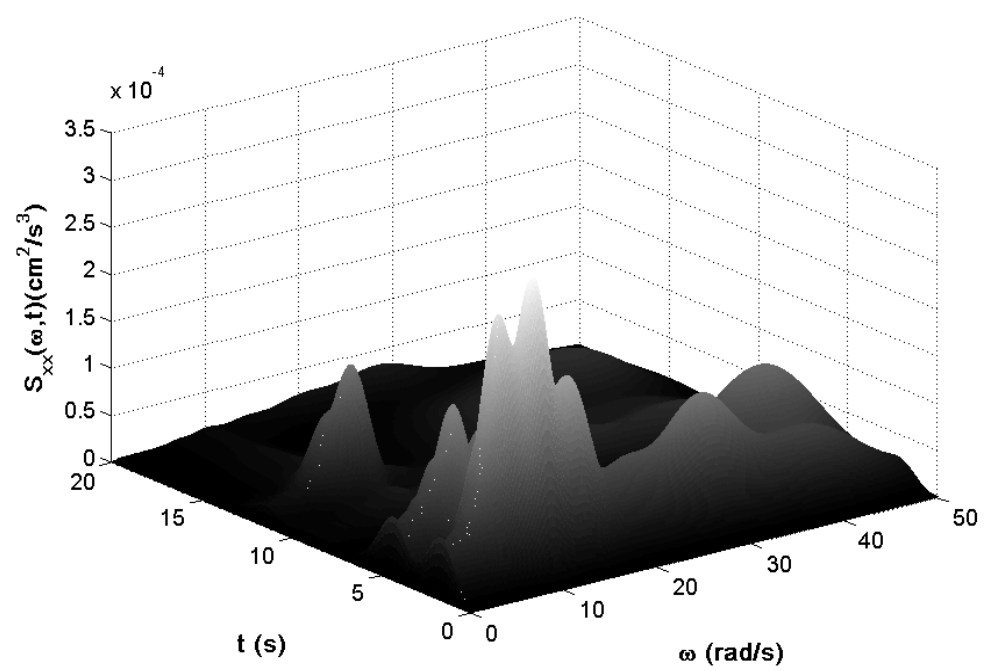

Fig. 9 Displacement PSDF using Morlet basis function for $\omega_{n}=40 \mathrm{rad} / \mathrm{s}$ and $\eta=10 \%$ for El Centro excitations.

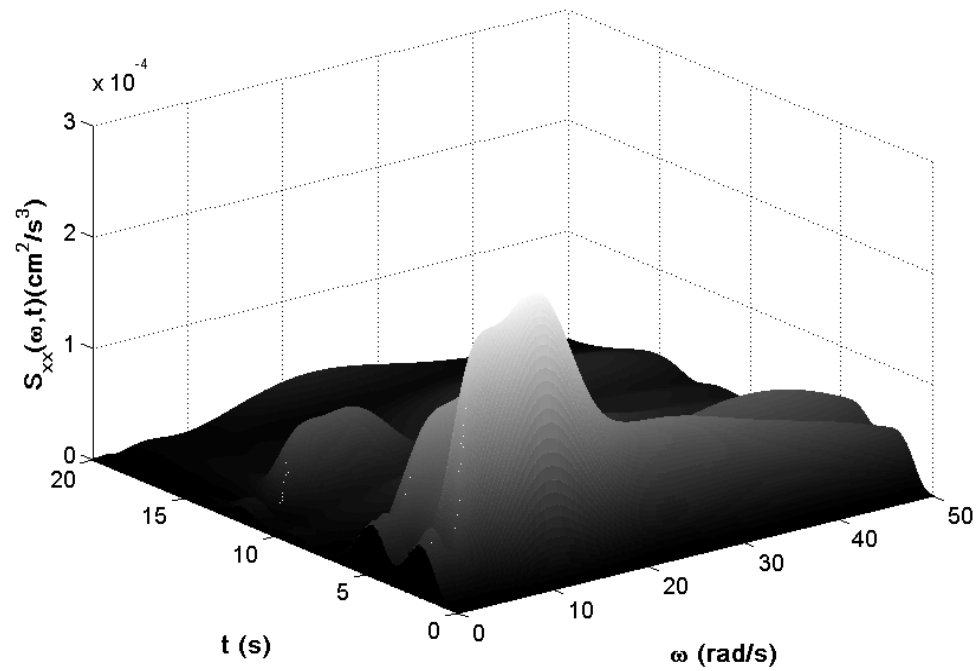

Fig. 10 Displacement PSDF using Mexican Hat basis function for $\omega_{n}=40 \mathrm{rad} / \mathrm{s}$ and $\eta=10 \%$ for El Centro excitations. 


\section{Conclusions}

The non-stationary response of a Duffing oscillator is evaluated using combined statistical linearization and continuous wavelet transform. As amplitude modulated stationary process is used at input, the parameters of the equivalent linear system vary with time. These parameters are evaluated by minimizing the mean square error between the displacements of the nonlinear system and the LTVS in wavelet domain. In this context, the input-output relation of a LTVS under non-stationary input is developed using time localization characteristics of wavelet transform, which is the uniqueness of the present work. The time-frequency analysis does not impose any restriction on the choice of the basis function. Three different basis functions are used to evaluate the displacement PSDF of the nonlinear system which matches closely with the simulation results. Moreover, as the linearization scheme is developed using the wavelet coefficients of the input process, the proposed linearization technique is applicable for either summation of amplitude modulated stationary orthogonal process or digitally simulated signals, which makes it more general. All these prove the efficiency and accuracy of the proposed wavelet based linearization to evaluate the stochastic response of the Dufffing type oscillator.

\section{References}

[1] D.E. Newland, An Introduction to Random Vibration, Spectral and Wavelet Analysis, Longman Scientific and Technical, Essex, 1983.

[2] N. Nigam, S. Narayanan, Application of Random Vibrations, Narosa Publishing House, New Delhi, 1994.

[3] L. Lutes, S. Sarkani, Random Vibrations, Butterworth-Heinemann Burlington, UK, 2004.

[4] T. Caughey, Derivation and application of Fokker-Planck equation to discrete dynamics system subjected to white random excitation, Journal of Acoustic Society of America 35 (1963) 1683-1692.

[5] T. Caughey, Non-linear theory of random vibrations, Advanced Applied Mechanics 11 (1971) 209-253.

[6] J. Hammond, Evolutionary spectra in random vibrations,
Journal of the Royal Statistical Society 35 (1973) 167-188.

[7] A.G. Orabi, A functional series expansion method for response analysis of nonlinear systems subjected to random excitations, Journal of Non-linear Mechanics 22 (1987) 451-465.

[8] E. Cross, K. Worden, Approximation of the Duffing oscillator frequency response function using the FPK equation, Journal of Sound and Vibration 330 (2011) 743-756.

[9] S. Crandall, On using non-Gaussian distributions to perform statistical linearization, International Journal of Non-linear Mechanics 39 (2004) 1395-1406.

[10] J.R. Booton, The analysis of nonlinear control system with random inputs, in: Proceedings of the Symposium on Nonlinear Circuit Analysis, Polytechnic Institute of Brooklyn, 1953, pp. 369-397.

[11] T. Caughey, Equivalent linearization techniques, Journal of the Acoustical Society of America 35 (1963) 1706-1711.

[12] W. Iwan, A. Mason, Equivalent linearization for systems subjected to non-stationary random excitation, International Journal of Non-linear Mechanics 35 (1980) 71-82.

[13] R.N. Iyengar, Stochastic response and stability of the Dufffing oscillator under narrow band excitation, Journal of Sound and Vibration 126 (2) (1988) 255-263.

[14] J. Roberts, P. Spanos, Random Vibration and Statistical Linearization, John Wiley and Sons, New York, 1990.

[15] I. Elishakoff, X.T. Zhang, An appraisal of different stochastic linearization criteria, Journal of Sound and Vibration 153 (1992) 370-375.

[16] I. Elishakoff, Multiple combinations of the stochastic linearization criteria by the moment approach, Journal of Sound and Vibration 237 (3) (2000) 550-559.

[17] C. Sobiechowski, L. Socha, Statistical linearization of the Duffing oscillator under non-Gaussian external excitation, Journal of Sound and Vibration 231 (2000) 19-35.

[18] H.J. Pradlwarter, Non-linear stochastic response distributions by local statistical linearization, International Journal of Non-linear Mechanics 36 (2001) 1135-1151.

[19] R. Mickens, A combined equivalent linearization and averaging perturbation method for non-linear oscillator equations, Journal of Sound and Vibration 264 (2003) 1195-1200.

[20] D. Roy, R. Kumar, A multi-step transversal linearization (MTL) method in non-linear structural dynamics, Journal of Sound and Vibration 287 (2005) 203-226.

[21] S. Lacquaniti, G. Ricciardi, Aprobabilistic linearization method for non-linear systems subjected to additive and 


\section{Oscillator Using Continuous Wavelet Transform}

multiplicative excitations, International Journal of Non-linear Mechanics 41 (2006) 1191-1205.

[22] W. Li, C. Chang, S. Tseng, The linearization method based on the equivalence of dissipated energies for nonlinearly damped structural systems, Journal of Sound and Vibration 295 (2006) 797-809.

[23] B. Basu, V.K. Gupta, Non-stationary seismic response of MDOF systems by wavelet transforms, Earthquake Engineering and Structural Dynamics 26 (1997) 1243-1258.

[24] B. Basu, V.K. Gupta, Seismic response of SDOF system by wavelet modeling of non-stationary processes, Journal of Engineering Mechanics 124 (10) (1998) $1142-1150$.

[25] B. Basu, V.K. Gupta, Stochastic seismic response of SDOF systems through wavelets, Engineering Structures 22 (2000) 1714-1722.

[26] B. Basu, V.K. Gupta, Wavelet based stochastic seismic response of a Duffing oscillator, Journal of Sound and Vibration 245 (2001) 251-260.

[27] A. Chakraborty, B. Basu, Non-stationary response analysis of long span bridges under spatially varying differential support motions using continuous wavelet transform, ASCE Journal of Engineering Mechanics 134 (2) (2008) 152-162.

[28] P. Spanos, G. Failla, Evolutionary spectral estimation using wavelets, Journal of Engineering Mechanics 130 (2004) 952-960.

[29] R. Rao, A. Bopardikar, Wavelet Transforms, Introduction to Theory and Applications, Addison Wesley Longman, Massachusetts, 1995.

[30] Y.T. Chan, Wavelet Basics, Kluwer Academic Publishers, Boston, 1995.

[31] M.B. Priestley, Power spectral analysis of non-stationary random processes, Journal of Sound and Vibration 6 (1967) 86-97.

[32] M.B. Priestley, Evolutionary spectra and non-stationary processes, Journal of the Royal Statistical Society, Series B 27 (1965) 204-237.

[33] J. Conte, B. Peng, Fully nonstationary analytical earthquake ground-motion model, Journal of Engineering Mechanics 123 (1997) 15-24. 